九州大学学術情報リポジトリ

Kyushu University Institutional Repository

\title{
Unbiased estimation based on sufficient statistics
}

Washio, Yasutoshi

Kyushu University

Morimoto, Haruki

Tokyo University

Ikeda, Nobuyuk i

Kyushu University

https://doi.org/10.5109/12969

出版情報: 統計数理研究. 6 (3/4)，pp.69-93，1956-03. Research Association of Statistical Sciences

バージョン :

権利関係 : 


\title{
UNBIASED ESTIMATION BASED \\ ON SUFFICIENT STATISTICS
}

\author{
By \\ Yasutoshi Washio, Haruki Morimoto and Nobuyuki IKEDA
}

(Received March 10, 1956)

\$1. Introduction. This paper treats the unbiased estimation under existence of sufficient statistics. Dealing with this problem, existence and uniqueness of unbiased estimates depending only on the sufficient statistics for the unknown parameters themselves and for a few of functions of the unknown parameters of normal distributions have been shown under some conditions by various authors like RAO [17], HoEL [10] and KOLMOGOROFF [12].

The main object of this paper is to show these results for a fairy general class of functions of the unknown parameters and derive the estimates explicitly when the distribution in question admits the sufficient statistics. General formulation of the problem is given in Section 2, on the basis of the following facts utilized by LeHMANn-Scheffé [14], GiRshick-SAVAGE [8], and others: (i) in our situation we may restrict our attention to the class of estimates depending only on the sufficient statistics and (ii) the distribution admitting the sufficient statistics is of so-called exponential type introluced by Koopman [13], Pitman [15] and Doob [5]. Then in Section 3 we will derive the unique unbiased sufficient estimate for a given parametric function and review about it from some operator-theoretic standpoint.' From the uniqueness of these estimates it follows that they are the best unbiased estimates in the sense that they minimize the expected loss with respect to any loss function (for example, variance) among all unbiased estimates. Section 4 exposes the applications to the cases of normal and gamma distributions. Variances of our estimates are treated in Section 5. Extension of these results to the case where the number of unknown parameters is more than one is discussed in Section 6 and some concrete examples are given in Section 7. Further, we will mention briefly the treatment of discrete case in Section 8. Some general aspects of operational methods hold also in these two cases treated in Sections 6 and 8 .

In preparation of this paper, the authors are much indebted to Prof. T. KITAGAWA for suggestions and encouragements.

§2. Statement of the problem. Let $X=\left(X_{1}, X_{2}, \ldots, X_{n}\right)$ be a random variable with cumulative distribution function $F(x ; \tau)$, where $x=\left(x_{1}\right.$,

1) We owe to Prof. T. Kitagawa's suggestion this operator-theoretic argument. Concerning to this cf. KITAGAWA[11]. 
$\left.x_{2}, \ldots, x_{n}\right)$ is any fixed point in $n$-dimensional euclidean space $R_{n}$ and $\tau$ is a real unknown parameter. Our problem is to estimate the value of some given function $\theta(\tau)$ of $\tau$ on the basis of observations on $X$.

By an estimate $\delta(X)$ we mean a statistical procedure which associates with each $x$ a probability measure $\delta(x)$ on $R_{1}$. An estimate is said to be non-randomized if for every $x \delta(x)$ assigns probability one to a single point of $R_{1}$. We denote by $L(\theta, a)$ the loss which results from making the estimate $a$ when $\theta$ is the true value of $\theta(\tau)$ and assume it to be a measurable function of $\theta$ and $a$. Our aim is to determine $\delta$ making the expected loss $r(\theta(\tau)$, $\delta(X))=\int L(\theta(\tau), \delta(\boldsymbol{x})) d F(x ; \tau)$ as small as possible. ${ }^{2)}$

Because of the difficulty of finding from the totality of the estimates the one which minimizes the expected loss for all $\tau$, it is ordinary to impose some natural conditions upon the estimete. Among these, unbiasedness is regarded one of the appealing conditions, and the theory of estimation so far has been mainly concerned with the best unbiased estimates. ${ }^{3)}$ In this paper we will confine ourselves to the unbiased estimates and among them seek for the one which minimizes the expected loss. Hence the classical problem of "minimum variance unbiased estimation" is a special case of our situation where $L(\theta, a)=(\theta-a)^{2}$.

For such statistical problems, the notion of sufficiency introduced by Fisher [7] plays an important rôle.") .First we give

Lemma 1. In any estimation problem, the class of all sufficient estimates, i.e., the estimates depending only on the sufficient statistic, is essentially complete.

To be concrete, assume that the distribution of $X$ admits the sufficient statistic $U$. Let $\theta(\tau)$ be a real-valued function of unknown parameter $\tau$, $\delta(X)$ be a given estimate of $\theta(\tau)$, and $r(\theta(\tau), \delta(X))$ be the expected loss resulted from any loss function. Then there exists an estimate $\delta^{\prime}(U)$ depending only on the sufficient statistic $U$ such that

$$
\boldsymbol{r}\left(\theta(\tau), \delta^{\prime}(U)\right) \leqq \boldsymbol{r}(\theta(\tau), \delta(X))
$$

for all $\tau$.

Proof. $\delta^{\prime}(U)=E[\delta(X) U]$ has the above-mentioned property.

This lemma is a slightly modified form of the theorem due to BLACKwELLGiRshick [2], and also an extension of the one known as "the theorem of RAO [16] and BLACKWELL [1]".

It is to be noted that in the above, if $\delta(X)$ is unbiased, so is $\delta^{\prime}(U)$. Therefore, if the distribution admits the sufficient statistic, in constructing the unbiased estimate we can restrict ourselves to the class of estimates

2) This formulation is essentially due to WALD's statistical decision theory. Cf. WALD [19].

3) For the notion of unbiasedness, see CRAMÉr [4].

4) Mathematical formulation and main properties of sufficiency are found in HaLmos-SAvaGe [9] and LEHMANN-SCHEFFÉ [14]. 
depending only on it. We may remark also that $\delta^{\prime}(U)$ is a non-randomized estimate. Therefore in any estimation problem, randomization can be eliminated under existence of sufficient statistic without any condition on the loss function, the parameter space, and etc.".)

Then the question arises what sort of distribution admits the sufficient statistics and how can we find these statistics. Answering to this, we will put forth a lemma which is due to Koopman [13], Pitman [15] and LEHMANN-SCHEFFÉ [14].

Lemma 2. Assume that $F_{0}(x ; \tau)$ can be written in the form

$$
d F_{0}(x ; \tau)=f_{0}(x ; \tau) d x=A_{0}(\tau) e^{B(\tau) u(x)+v_{0}(x)} d x,
$$

and the set $\mathfrak{D}$ on which $f_{0}(x ; \tau) \neq 0$ is independent of $\tau$ (except possibly a set of measure zero). Then, $U=u(X)$ is a sufficient statistic for this class of distributions.

Conversely, assume that $F_{0}(x ; \tau)$ admits a sufficient statistic, and satisfies the above condition concerning $\mathcal{D}$. And if the derivative $\frac{\partial^{*} f_{0}}{\partial \boldsymbol{x} \partial \tau}$ exists for almost all $x$ and for all $\tau$, it can be written in the form (2.1).

In virtue of these two lemmas we may and we shall restrict ourselves to sufficient non-randomized estimates and treat the estimation problem about the distribution of the form (2.1). Further, we will treat it about the distribution of the form $A_{0}(\tau) e^{-\tau u^{(}(x)+v_{0}(x)}$ only, without loss of generality for any estimation problem.

\$3. Unbiased sufficient estimates. Let the random variable $X=$ $\left(X_{1}, X_{2}, \ldots, X_{n}\right)$ have a generalized probability density ${ }^{6)}$

$$
A_{0}(\tau) e^{-\tau u(x)+v_{\|}(x)}
$$

with respect to a measure $\mu$ on $\boldsymbol{R}_{n}$, where $u(\boldsymbol{x})$ and $v_{v}(\boldsymbol{x})$ are real valued measurable functions, and $\tau$ ranges over a non-empty set $T$ of real numbers, on which

$$
\left\{A_{v}(\tau)\right\}^{-1}=\int_{R_{n}} e^{-\tau u(x)+v_{\|}(x)} d \mu(x)<\infty .
$$

Then $U=u(X)$ is a sufficient statistic for this family of probability distributions with a generalized probability density

$$
A(\tau) e^{-\tau u}
$$

with respect to a measure $\nu(u)$ on $R_{1}$, where $u$ is real and for $\tau \in T$,

$$
\{A(\tau)\}^{-1}=\int_{-\infty}^{\infty} e^{-\tau u} d \nu(u)<\infty .
$$

5) Cf. Dvoretzkx-Waid-Wothowitz [6] and BLackwheli-Girshick [2].

6) With respect to the concept of gencralized probability density, sec LEHMAN-SCHEFFE [1]]. 
Suppose that $T^{\prime}$ is a non-degenerated interval such that $T \subset T^{\prime}$, and the integral

$$
\int_{-\infty}^{\infty} u e^{-\tau u} d \nu(u)
$$

converges for all $\tau \in T^{\prime}$. Let $Z$ be the set of all complex numbers $z=\tau+i t$ for which $\tau \in T$, then it is seen that $\{A(z)\}^{-1}$ is analytic for $z \in Z{ }^{i}$ )

In this and subsequent Sections $(\S 3 \sim \S 4)$, we shall assume that the above-mentioned measure $\nu(u)$ is absolutely continuous with respect to the Lebesgue measure on $R_{1}$, that is, $U$ has a probability density $f(u ; \tau)$ which is written in the form

$$
f(u ; \tau)=A(\tau) e^{-\tau u+v(u)},
$$

where the carrier $\mathfrak{I}(u)$ of $e^{v(u)}$ is independent of $\tau$.

From these considerations we will give the following

Theorem 1. Let $U$ have the probability density of the form (3.5). Assume that the following conditions are satisfied:

(i) $\theta(z)$ is analytic in the strip such that $s \in T$, where $z=s+i$,

$$
\int_{-\infty}^{\infty}\left|\varphi_{s}(-t) \theta(s+i t)\right| d t<\infty, \quad \text { for } s \in T \text {, }
$$

where $\varphi_{s}(t)$ is the characteristic function of the distribution of $U$, when $\tau=\boldsymbol{s}$,

$$
\lim _{t \mid \rightarrow \infty} \varphi_{s}(-t) \theta(s+i t)=0
$$

uniformly in every closed subinterval of $s \in T$.

Then if we put

$$
\begin{aligned}
& w(u)=\frac{1}{2 \pi i} \int_{s-i \infty}^{s+i \infty} \frac{\theta(z)}{A(z)} e^{z u} d z \quad(s \in T, u \in \mathfrak{D}(u)), \\
& \delta(U)=e^{-v(u)} w(u)_{u=r}^{\text {s }}
\end{aligned}
$$

is the unique unbiased sufficient estimate of $\theta(\tau) .^{n)}$

Further, if there exists a linear operator $\Lambda_{n}$ satisfying the condition ${ }^{(1)}$

$$
\Lambda_{u} e^{\tau u}=\theta(\tau) e^{\tau u}
$$

and existence of both members and equality

$$
\int_{-\infty}^{\infty} A_{u}\left\{\frac{e^{(s+i t) u}}{A(s+i t)}\right\} d t=A_{u}\left\{\int_{-\infty}^{\infty} \frac{e^{(s+i t) u}}{A(s+i t)} d t\right\}
$$

7) Cf. SverDRup [18], § 2 .

8) The symbol "| $u=l$ " means substitution of $U$ for $u$.

9) Here the term "unique" is to be comprehended in the sense of "unique at almost every. where."

10) Cf. Kitaliawa[11], Theorem 3.1. 
are affirmed, then the above estimate can be expressed in the form

$$
\delta(U)=\left.e^{-v(u)} A_{u} e^{v(u)}\right|_{u=l} .
$$

Proof. Since for $z \in Z A(z)$ is analytic it is seen that from the convergence of $(3.4)$

$$
\{A(s+i t)\}^{-1}=\frac{1}{A(s)} \int_{\mathscr{D}(u)} e^{-i t u}\left\{A(s) e^{\left.-s u+v^{\prime} u\right)}\right\} d u=\varphi_{s}(-t)\{A(s)\}^{-1} .
$$

Making use of the theorem concerning Laplace transforms we have by the conditions (i), (ii) and (iii) given above ${ }^{(1)}$

$$
\theta(\tau)=A(\tau) \int_{\mathfrak{O}(u)} e^{-\tau u} w(u) d u, \quad \tau \in T
$$

Hence

$$
\theta(\tau)=\int_{\mathcal{D}(u)} \delta(u)\left\{A(\tau) e^{-\tau u+v(u)}\right\} d u
$$

If we use (iv), $w(u)$ becomes by (v)

$$
w(u)=\frac{1}{2 \pi i} \int_{s-i \infty}^{s+i \infty} \frac{\Lambda_{u} e^{2 u}}{A(z)} d z=\Lambda_{u}\left\{\frac{1}{2 \pi i} \int_{s-i \infty}^{s+i \infty} \frac{e^{z u}}{A(z)} d z\right\}=\Lambda_{u} e^{v(u)}
$$

Hence

$$
\delta(u)=e^{-v(u)} A_{u} e^{v \cdot u)} .
$$

Uniqueness of estimate is also due to the corresponding property of Laplace transforms. q.e.d.

It is to be noted here that the conditions (ii) and (iii) of Theorem 1 are equivalent to the following two conditions (ii)' and (iii) ${ }^{\prime}$ respectively:

$(\text { ii })^{\prime}$

$$
\begin{aligned}
& \int_{-\infty}^{\infty}\left|\frac{\theta(s+i t)}{A(s+i t)}\right| d t<\infty, \quad \text { for } s \in T, \\
& \lim _{t \rightarrow \infty} \frac{\theta(s+i t)}{A(s+i t)}=0
\end{aligned}
$$

uniformly in every closed subinterval of $s \in T$.

Remark 1. Let $\delta_{k}(U)$ be the unique unbiased sufficient estimate of $\theta_{k}(\tau)(k=0,1,2, \ldots)$. If the conditions in Theorem 1 imposed on $\theta(\tau)$ are satisfied by all $\theta_{k}(\tau)$ 's, and if

$$
\theta_{u}(s+i t)=\sum_{k=1}^{\infty} a_{k} \theta_{k}(s+i t),
$$

11) Cf. Widder [20], p. 265. 
uniformly in $t$ for any fixed $s \in T$, where $a_{k}$ are constants, then

$$
\delta_{0}(U)=\sum_{k=1}^{\infty} a_{k} \delta_{k}(U)
$$

almost everywhere for all $\tau$. This is an immediate consequence of the calculations in the proof of Theorem 1.

The assumptions above seem to be inevitable, but in most cases they are easily verified. For example reader may refer to $\S 4$, where applications to normal and gamma distributions are treated.

Further, it is to be noted that the operator-theoretic terms employed in the latter part of Theorem 1 are useful in some cases for simplifying our arguments. Looking for the unbiased estimate of $\theta(\tau)$, it suffices us to find the operator $A_{u}$, of which $\theta(\tau)$ is the generating function. Following results can be got along this line.

Corollary 1.1. Assume that the sufficient statistic $U$ has the probability density of the form (3.5) and for an assigned non-negative integer $k f(u ; \tau)$ has first $(k+1)$ derivatives with respect to $u$ all belonging to the class $L_{1}(-\infty, \infty)$. Then if the condition (iii) of Theorem 1 is satisfied, the unique unbiased sufficient estimate of $\theta(\tau)=\tau^{k}$ is

$$
\delta(U)=\left.e^{-v(u)} D^{k} e^{v(u)}\right|_{u=c}=\left.e^{-v(u)} \frac{\partial^{k}}{\partial u^{k}} e^{v(u)}\right|_{u=c^{k}} ^{{ }^{12)}}
$$

Proof. Since the conditions (i) and (ii) of Theorem 1 are satisfied, that the operator $A_{u}$ is given by

$$
\Lambda_{u} \boldsymbol{g}(\boldsymbol{u})=\frac{\partial^{k}}{\partial \boldsymbol{u}^{k}} \boldsymbol{g}(\boldsymbol{u})
$$

is clear.

This result together with Remark 1 above enables us to estimate any function $\theta(\tau)$ which can be expanded in the form $\theta(\tau)=\sum_{k=0}^{\infty} a_{k} \tau^{k}$ provided that the above conditions are fulfilled.

Corollary 1.2. Assume that sufficient statistic $U$ has the probability density of the form (3.5), and $T=(0, \infty)$. Let

$$
\theta(\tau)=\tau^{-\alpha} \quad(\alpha \text { is a positive real number })
$$

together with the probatility density $f(u ; \tau)$ satisfies the conditions (ii) and (iii) of Theorem 1. Then the unique unbiased sufficient estimate $\delta(U)$ of $\theta(\tau)$ is given by

$$
\delta(U)=\left.e^{-v(u)} D^{-\alpha} e^{v(u)}\right|_{u=c}=\left.e^{-v(u)} \frac{1}{\Gamma(\alpha)} \int_{0}^{\infty} x^{\alpha-1} e^{v(u-x)} d x\right|_{u=v} .
$$

12) For every real number $\alpha$ the symbol " $\left.D^{\alpha\{}\right\}$ " means integral of fractional order $\alpha$ if $\alpha<0$ and derivative of fractional order $\alpha$ if $\alpha \geqq 0$. Cf. Zygmund [21]. See also Kitagawa [11], Example 3.1. 
Corollary 1.3. Let $U$ have the probability density of the form (3.5), and $T=(0, \infty)$. Assume that for any positive real number $\alpha$ the probability density of $U$ has first $([\alpha]+2)$ derivatives with respect to $u$ all belonging to the class $L_{1}(-\infty, \infty)$. ${ }^{1: 3}$

Then, if the function of the unknown parameter $\tau$

$$
\theta(\tau)=\tau^{\alpha}
$$

satisfies the condition (iii) of Theorem 1, its unique unbiased sufficient estimate $\delta(U)$ is given by

$$
\delta(U)=\left.e^{-v(u)} D^{\alpha} e^{v(u)}\right|_{u=c}=\left.\left.e^{-v(u)} \frac{1}{\Gamma(m-\alpha)} \frac{\partial^{m}}{\partial u^{m}}\right|_{0} ^{\infty} x^{m-\alpha-1} e^{v(u-x)} d x\right|_{u=v},
$$

where $m=[\alpha]+1$.

Proof. It can be easily verified that the conditions (i) and (ii) of Theorem 1 are satisfied. Then the determination of the operator $A_{u}$ is quite immediate.

Corollary 1.4. Let $U$ have the probability density of the form (3.5). Assume that for an assigned complex number $c$,

$$
\theta(\tau)=e^{c \tau}
$$

satisfies conditions (ii) and (iii) of Theorem 1. If $v(u)$ is defined for every complex value of $u$, we obtain the unique unbiased sufficient estimate $\delta(U)$ of $\theta(\tau)$ by the following:

$$
\delta(U)=e^{-v(\tau)+v(l+c)} .
$$

§4. Applications (1). In this Section we will give some concrete examples playing important rôles in the statistical inference, to illustrate that the conditions given in $\S 3$ are actually satisfied, and to obtain the unbiased estimates in more explicit form.

Example 1. The case where the sufficient statistic is distributed according to a normal distribution.

When $X_{i}$ 's $(i=1,2, \ldots, n)$ are distributed independently in $N(\tau, 1)$, $\tau$ being the unknown mean value, $-\infty<\tau<\infty$, the distribution of $X=\left(X_{1}\right.$, $\left.X_{2}, \ldots, X_{n}\right)$ is written in the form

$$
\left(\frac{1}{\sqrt{2 \pi}}\right)^{n} e^{-\frac{n}{2} \tau^{2}} e^{-\tau_{1}\left(-\sum_{i=1}^{n} x_{i}\right)-\frac{1}{2} \sum_{i=1}^{n} x_{i}{ }^{2}}
$$

13) "[ ]" is Gauss" symbol. 
Then $U=u(X)=-\sum_{i=1}^{n} X_{i}$ is the sufficient statistic for this class of distributions and its probability density and characteristic function are

$$
\frac{1}{\sqrt{2 \pi n}} e^{-\frac{n}{2} \tau^{2}} e^{-\tau u-\frac{u^{2}}{2 n}}
$$

and

respectively.

$$
\varphi_{\delta}(t)=e^{-i n s t-\frac{1}{2} n t^{2}}
$$

Problem 1. 1. Consider the function $\theta(\tau)=\tau^{k}, k$ being a non-negative integer.

Here the conditions in Corollary 1.1 are obviously satisfied. The unique unbiased sufficient estimate $\delta(U)$ of $\theta(\tau)$ is given by

$$
\delta(U)=\left(-\frac{1}{\sqrt{n}}\right)^{k} H_{k}\left(\frac{U}{\sqrt{ } \frac{}{n}}\right)
$$

where $H_{k}(x)$ are Hermite polynomials, i.e.,

$$
H_{k}(x)=(-1) e^{k} \frac{x^{2}}{\partial x^{k}} e^{-\frac{x^{2}}{2}}
$$

This result obtained for Problem 1.1 together with Remark 1 in Section 3 shows that in this case the unique unbiased sufficient estimate of an $\boldsymbol{n}$-th order polynomial of $\tau$ is also an $n$-th order polynomial of $X$. Furthermore, the $n$-th order coefficients of both polynomials coincide.

Problem 1.2. For a given complex value of $c$, the unique unbiased sufficient estimate $\delta(U)$ of

$$
\theta(\tau)=e^{c \tau}
$$

is, from Corollary 1.4, given by

$$
\delta(U)=e^{-v(U)+v\left(l^{+}+c\right)}=e^{\frac{l^{2}}{2 n}-\frac{(l+c)^{2}}{2 n}}=e^{-\frac{c^{2}}{2 n}-\frac{c l^{\prime}}{n}} .
$$

Problem 1.3. Suppose that for $a<n$,

$$
\theta(\tau)=e^{-\frac{a}{2}(\tau-b)^{2}} .
$$

To this problem Theorem 1 is applicable. Therefore we have

$$
\begin{aligned}
w(u) & =\frac{1}{2 \pi i} \int_{-i \infty}^{i \infty} e^{-\frac{a}{2}(z-b)^{2}}\left\{\sqrt{2 \pi n} e^{-\frac{n z^{2}}{2}}\right\} e^{z u} d z \\
& =\frac{\sqrt{n}}{\sqrt{2 \pi}} e^{-\frac{a}{2} b^{2}} \int_{-\infty}^{\infty} e^{-i t u} e^{-\frac{a t}{2}(n-a) t^{2}+i a u t} d t \\
& =\frac{\sqrt{ } n}{\sqrt{n-a}} e^{-\frac{a b^{2}}{2}} e^{-\frac{(u+a b)^{2}}{2(n-a)}} .
\end{aligned}
$$


Hence

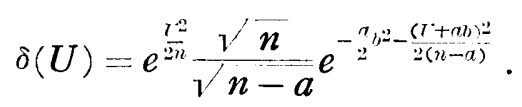

Especially if we put $a=1$ and $b=y$, where $y$ is an assigned real number, it is possible to estimate the probability density of $N(\tau, 1)$ at a given point $y$, on the basis of the independent sample of size $n(n>1)$. Thus as the unique unbiased sufficient estimate of

$$
\frac{1}{2 \pi} e^{-\frac{1}{2}(y-\tau)^{2}}
$$

we obtain

$$
\frac{1}{\sqrt{2 \pi\left(1-\frac{1}{n}\right)}} e^{-\frac{(y-\bar{x})^{2}}{2(1-1 / n)}} \quad(n>1)
$$

Next, we are to estimate the probability that values of independent sample of size $k$ all belong to a set $A$ simultaneously, based on a observation of size $n(n>k)$ which is independent of the sample in question. Namely, suppose that

$$
\theta(\tau)=\left[\frac{1}{\sqrt{ } 2 \pi} \int_{A} e^{-\frac{(y-\tau)^{2}}{2}} d y\right]^{k}
$$

Then we have

$$
\theta(\tau)=\left(\frac{1}{\sqrt{2 \pi}}\right)^{k} \int_{A} \ldots \int_{A} e^{-\frac{1}{2} \sum_{i=1}^{k} y_{i}{ }^{2}+\frac{1}{2} k\left(\frac{1}{k} \sum_{i=1}^{k} y_{i}\right)^{2}} e^{-\frac{1}{2} k\left[\tau-\frac{1}{k} \sum_{i=1}^{k} y_{i}\right]^{2}} d y_{1} \ldots d y_{k},
$$

Making use of (4.4) in the case where $a=k$ and $b=1 / k \sum_{i=1}^{k} y_{i}$ we obtain the unique unbiased sufficient estimate of $\theta(\tau)$ by

$$
\begin{aligned}
\delta(U) & =\left(\frac{1}{\sqrt{2 \pi}}\right)^{k} \frac{\sqrt{n}}{\sqrt{n-k}} e^{\frac{l^{2}}{2 n}} \int_{A} \ldots \int_{A} e^{-\frac{1}{2} \sum_{i=1}^{k} y_{i^{2}-\frac{1}{2}} \frac{\left(l+\sum_{i=1}^{k} y_{i}\right)^{2}}{(n-k)}} d y_{1} \ldots d y_{k} \\
& =\left(\frac{1}{\sqrt{2 \pi}}\right)^{k} \frac{\sqrt{n}}{\sqrt{n-k}} \int_{A} \ldots \int_{A} e^{-\frac{n-k+1}{2(n-\bar{k})} \sum_{i, j=1}^{k} a_{i j}\left(y_{i}-\bar{x}\right)\left(y_{j}-\bar{x}\right)} d y_{1} \ldots d y_{k},
\end{aligned}
$$

where

$$
a_{i j}=\left\{\begin{array}{cl}
1 & i=j \\
\frac{1}{n-k+1} & i \neq j .
\end{array}\right.
$$

In the case where $k=1$, this result coincides with the one obtained by KOLMOGOROFF [12]. ${ }^{14)}$

Example 2. The case where the sufficient statistic is distributed according to the gamma distribution.

14) The idea due to Prof. T. Kitaciawa made the unified treatment of the problem for $k=1$ and $k>1$ possible. 
Then the probability density of $U=u(X)$ is

$$
\frac{\tau^{m}}{\Gamma(m)} e^{-\tau u} u^{m-1}
$$

where $m$ is an assigned positive number, $\tau>0$ and $0<u<\infty$.

That for a given value of $\alpha<\boldsymbol{m} \quad \theta(\tau)=\tau^{\alpha}$ satisfies the conditions of Corollary 1.3 is quite evident. Then the unique unbiased sufficient estimate $\delta(U)$ of $\tau^{\alpha}$ is by Corollary 1.3

$$
\delta(U)=\frac{\Gamma(m)}{\Gamma(m-\alpha)} U^{-\alpha} .
$$

Problem 2.1. Suppose that the random variables $X_{i}(i=1,2, \ldots, n)$ are $n$ identically distributed independent normal variables with mean 0 and unknown variance $\sigma^{2}$. Then the probability density of $X=\left(X_{1}, X_{2}, \ldots\right.$, $\left.X_{n}\right)$ is

$$
\left(\frac{1}{\sqrt{2 \pi}}\right)^{n} \frac{1}{\sigma^{n}} e^{-\frac{\sum_{i=1}^{n} x_{i}{ }^{2}}{2 \cdot \sigma^{2}}} .
$$

Putting $u(x)=\sum_{i=1}^{n} x_{i}^{2}, \tau=\frac{1}{2 \sigma^{2}}$, we observe that $U=u(X)$ is the sufficient statistic for this family of distributions and has the probability density

$$
\frac{1}{2^{n / 2} \Gamma(n / 2)} \frac{1}{\sigma^{n}} e^{-\frac{1}{2 \sigma^{2}} u} u^{\frac{n}{2}-1} \text {. }
$$

So that we obtain for $\theta(\tau)=\tau^{-1} / 2\left(\equiv \sigma^{2}\right)$, the unique unbiased sufficient estimate

$$
\delta(U)=\frac{\Gamma(n / 2)}{\Gamma(n / 2+1)} U=\frac{1}{n} U \quad\left(\equiv \frac{\sum_{i=1}^{n} X_{i}^{2}}{n}\right),
$$

and for $\theta(\tau)=\tau^{-1 / 2} / 2 \quad(\equiv \sigma)$,

$$
\delta(U)=\frac{\Gamma\left(\frac{n}{2}\right)}{\sqrt{2} \Gamma\left(\frac{n}{2}+\frac{1}{2}\right)} \sqrt{\sum_{i=1}^{n} X_{i}^{2}} .
$$

Problem 2.2. Suppose that $X_{i}$ 's $(i=1,2, \ldots, n)$ are distributed according to the gamma distribution. That is, the probability density of $X=$ $\left(X_{1}, X_{2}, \ldots, X_{n}\right)$ is of the form

$$
\frac{\tau^{n \alpha}}{\{\Gamma(\alpha)\}^{n}} e^{-\tau\left(\sum_{i=1}^{n} x_{i}\right)} \cdot \prod_{i=1}^{n} x_{i}^{\alpha-1},
$$

where $\tau$ is a positive unknown parameter and $\alpha$ is an assigned positive 
constant. In this case $U \because \cdots(X)=\sum_{i=1}^{n} X_{i}$ is the sufficient statistic and its probability density is

$$
\Gamma(n \alpha) e^{\tau^{n \alpha}} e^{-\tau u} u^{n \alpha-1}
$$

So that for $\theta(\tau)=\tau^{\beta}$, where $\beta$ is a real number such that $\beta<\boldsymbol{n} \alpha$, we obtain the unique unbiased sufficient estimate

$$
\delta(U)=\frac{\Gamma(\boldsymbol{n} \alpha)}{\Gamma(\boldsymbol{n} \alpha-\beta)} U^{-\beta} .
$$

5. Variances of estimates. In this Section we will concern ourselves with the problem of calculating the variances of our estimates obtained in the previous Sections. If the functional forms of $f(x ; \tau)$ and $\theta(\tau)$ are given, we can mostly give the conditions for the existence of the variance explicitly. And in certain circumstances we can even give the variances themselves.

If the variance of our estimate $\delta(U)$ exists $\delta(U)$ is the minimum variance unbiased estimate of its expected value. 'Therefore, it is interesting to compare this with CRAMÉR-RAO's lower bound, which is not necessarily attainable by any estimate. For an example of the case where the variance of our estimate does not exist, we will show the following:

In Example 1 of Section 4, let

$$
\theta(\tau)=e^{\frac{n^{2}}{2(k-n)} \tau^{2}},
$$

where $k / 2<n<k$. By a simple calculation we have

$$
\frac{1}{\sqrt{ } 2 \pi n} \int_{-\infty}^{\infty} e^{\frac{u^{2}}{2 k}} e^{-\frac{(u+n \tau))^{2}}{2 n}} d u=\sqrt{\frac{k}{k-n}} e^{\frac{n^{2}}{2(k-n)^{2}}} .
$$

Therefore $\theta(\tau)$ has the unique unbiased sufficient estimate

$$
\sqrt{\frac{k-n}{k}} e^{\frac{c^{2}}{2 k}}
$$

and if $k / 2<n<k$ it is evident that the variance of our estimate does not exist. In such cases unbiased estimate of $\theta(\tau)$ with finite variance does not exists.

Now we will give the variances of estimates for a few cases.

Proposition 1. In Problem 1.1 of $\$ 4$, the variance of the unique unbiased sufficient estimate $\delta\left(U^{T}\right)=(-1 / \sqrt{n})^{k} H_{k}(U / \sqrt{n})$ of $\tau^{k}$ exists, and is given by

$$
V\{\delta(U)\}=\sum_{i=u}^{k}\left({ }_{k} C_{i} \sqrt{(k-i) !} \tau^{i}(\sqrt{n})^{i-k}\right)^{2}-\tau^{2 k}
$$


Proof. Existence of the variance of $\delta(U)$ is clear. First we calculate the covariance of the estimates $\delta_{k}(U)$ of $\tau^{k}$ and $\delta_{l}(U)$ of $\tau^{\prime}$. For $k \geqq l$,

$$
\begin{gathered}
(\sqrt{n})^{k+l} E\left\{\delta_{k}(U) \delta_{l}(U)\right\}=\int_{-\infty}^{\infty}\left\{H_{i}\left(\frac{u}{\sqrt{n}}\right) H_{l}\left(\frac{u}{\sqrt{n}}\right)\right\}\left\{\frac{1}{\sqrt{2 \pi n}} e^{-n_{2}^{n} \tau^{2}} e^{-\tau u-\frac{u^{2}}{2 n}}\right\} d u \\
=\frac{1}{\sqrt{2 \pi}} \int_{-\infty}^{\infty} H_{k}(u-\lambda) H_{l}(u-\lambda) e^{-\frac{u^{2}}{2}} d u,
\end{gathered}
$$

where $\lambda=\sqrt{n} \tau$. Then making use of relations

$$
\begin{aligned}
& H_{k}(u-\lambda)=\sum_{j=1}^{k} \frac{1}{j !}(-\lambda)^{j} H_{k}^{(j)}(u), \\
& H_{k}^{(j)}(u)=k(k-1) \ldots(k-j+1) H_{k-j}(u),
\end{aligned}
$$

where

$$
H_{k}^{(j)}(\boldsymbol{u})=\frac{\partial^{j}}{\partial u^{j}} H_{k}(u),
$$

we have

$$
\begin{aligned}
& =\frac{1}{\sqrt{2 \pi}} \int_{-\infty}^{\infty}\left\{\sum_{j=0}^{k} \frac{1}{j !}(-\lambda)^{j}{ }_{k} C_{j}(j !) H_{k-j}(u)\right\}\left\{\sum_{i=1}^{l} \frac{1}{i !}(-\lambda)^{i} C_{l}(i !) H_{l-i}(u)\right\} e^{-\frac{u^{2}}{2}} d u \\
& =\sum_{j=0}^{k} \sum_{i=0}^{l}{ }_{k} C_{j l} C_{i}(-\lambda)^{i+j} \frac{1}{\sqrt{2} \pi} \int_{-\infty}^{\infty} H_{k-j}(u) H_{l-i}(u) e^{-\frac{u^{2}}{2}} d u .
\end{aligned}
$$

By the orthogonality of Hermitean polynomials

$$
\frac{1}{\sqrt{2 \pi}} \int_{-\infty}^{\infty} H_{k-j}(u) H_{l-i}(u) e^{-\frac{u^{2}}{2}} d u=\left\{\begin{array}{cc}
(k-j) ! & k-j=l-i \\
0 & k-j \neq l-i
\end{array}\right.
$$

So that we obtain

$$
\begin{aligned}
E\left\{\delta_{l}(U) \delta_{k}(U)\right\}=(\sqrt{n})^{-k-l} & \sum_{i=0}^{l}{ }_{k} C_{k-l+i} \cdot{ }_{l} C_{i}(-\lambda)^{k-l+2 i}(l-i) ! \\
= & \sum_{i=0}^{l}{ }_{k} C_{k-l+i} \cdot{ }_{l} C_{i}(-\lambda)^{k-l+2 i}(l-i) ! .
\end{aligned}
$$

Putting $k=l$, we get the required result.

In this problem it is seen that CRAMÉR-RAO's lower bound is given by

$$
\frac{k^{2}}{n} \tau^{2 k-2}
$$

Hence the efficiency is unity if and only if $k=1$.

Proposition 2. In Problem 1.2 of $\S 4$, the variance of our estimate $\delta(U)$ $=e^{-\alpha^{2} /(2 n)-(\alpha l) / n}$ of $\theta(\tau)=e^{\alpha \tau}$ (where $\alpha$ is an assigned real number) esists, and is given by

$$
V\{\delta(U)\}=-\left(e^{\frac{\alpha^{2}}{n}}-1\right) e^{2 \tau \alpha}
$$


Proof. We have

$$
\begin{aligned}
& E\left\{\delta(U)^{:}\right\}=e^{-\frac{\alpha^{2}}{2 n}} \int_{0}^{\infty} e^{-\frac{\alpha}{n} u}\left\{\frac{1}{V} \frac{1}{2 \pi n} e^{-\frac{n \tau^{2}}{2}} e^{-\tau u-\frac{u^{2}}{2 n}}\right\} d u \\
& -e^{\frac{\alpha^{2}}{n}} \int_{1}^{\infty} e^{-\frac{(2 \alpha)^{2}-2 \alpha u}{2 n}-\frac{1}{n}}\left\{\frac{1}{\sqrt{2 \pi n}} e^{-\frac{n \tau^{2}}{2}} e^{-\tau u-\frac{u^{2}}{2 n}}\right\} d u \\
& =e^{\frac{\alpha^{2}}{11}} e^{2 \tau \alpha} .
\end{aligned}
$$

Hence our proposition is established.

The variance of this estimate is greater than CRAMÉR-RAO's lower bound $\alpha^{2} / n \cdot e^{2 \alpha \tau}$ for all $\alpha \neq 0$.

Proposition 3. In the problem 2.2 of $\$ 4$, the variance of our estimate $\delta(U)=\frac{\Gamma(\boldsymbol{n} \alpha)}{\Gamma(\boldsymbol{n} \alpha-\beta)} U^{-\beta}$ of $\theta(\tau)=\tau^{\beta}$ (where $\beta<\boldsymbol{n} \alpha$ ) exists, and is given by

$$
V\{\delta(U)\}=\left\{\left(\frac{\Gamma(n \alpha)}{\Gamma(n \alpha-\beta)}\right)^{2} \Gamma(n \alpha-2 \beta)+1\right\} \tau^{2 \beta}
$$

Proof. We have

$$
\begin{aligned}
& E\left\{\delta\left(\boldsymbol{U}^{2}\right)\right\}=\left(\frac{\Gamma(\boldsymbol{n} \alpha)}{\Gamma(\boldsymbol{n} \alpha-\beta)}\right)^{2} \int_{0}^{\infty} u^{-2 \beta}\left[\frac{\tau^{2 \alpha}}{\Gamma(\boldsymbol{n} \alpha)} u^{n \alpha-1} e^{-\tau u}\right] d u \\
& =\left(\frac{\Gamma(n \alpha)}{\Gamma(n \alpha-\beta)}\right)^{2} \frac{\Gamma(n \alpha-2 \beta)}{\Gamma(n \alpha) \tau^{-2 \beta}} . \quad \text { q.e.d. }
\end{aligned}
$$

In this case, CRAMÉR-RAO's lower bound is given by $\beta^{2} / n \alpha \cdot \tau^{2 \beta}$.

§ 6. Two unknown parameters. Generalization of $\S 2 \sim \S 5$ to the case of several unknown parameters is quite immediate. And so in this Section we will treat only a few important results in the case of two parameters.

Suppose that the random variable $X=\left(X_{1}, X_{2}, \ldots, X_{n}\right)$ has the generalized probability density

$$
A_{v}\left(\tau_{1}, \tau_{2}\right) e^{-\tau_{1} u_{1}(x)-\tau_{2} u_{2}(x)+v_{1}(x)}
$$

with respect to a measure $\mu$ on $R_{n}$, where $u_{1}(x), u_{2}(x)$ and $v_{0}(x)$ are real valued measurable functions, and $\tau_{1}$ and $\tau_{2}$ range over non-empty sets $T_{1}$ and $T_{2}$ of real numbers respectively, and on which

$$
\left\{A_{0}\left(\tau_{1}, \tau_{2}\right)\right\}^{-1}=\int e^{-\tau_{1} u_{1}(x)-\tau_{2} u_{2}(x)+v_{v}(x)} d \mu(x)<\infty .
$$

Then, $U=\left(U_{1}, U_{2}\right)=\left(u_{1}(X), u_{2}(X)\right)$ is a sufficient statistic for this family of distributions with a generalized probability density

$$
A\left(\tau_{1}, \tau_{2}\right) e^{-\tau_{1} u_{1}-\tau_{2} u_{2}}
$$


with respect to a measure $\nu\left(u_{1}, u_{2}\right)$ on $R_{2}$, where $u_{1}$ and $u_{2}$ are real and for $\tau_{1} \in T_{1}$ and $\tau_{2} \in T_{2}$,

$$
\left\{A\left(\tau_{1}, \tau_{2}\right)\right\}^{-1}=\int_{-\infty}^{\infty} \int_{-\infty}^{\infty} e^{-\tau_{1} u_{1}-\tau_{2} u_{2}} d \nu\left(u_{1}, u_{2}\right)<\infty .
$$

Suppose that $T_{1}^{\prime}$ and $T_{2}{ }^{\prime}$ are non-degenerated intervals such that $T_{1} \subset T_{1}^{\prime}$ and $T_{2} \subset T_{: \prime}^{\prime}$ and the integral

$$
\int_{-\infty}^{\infty} \int_{-\infty}^{\infty} u_{1} u_{2} e^{-\tau_{1} u_{1}-\tau_{2} u_{2}} d \nu\left(u_{1}, u_{2}\right)
$$

converges for all $\tau_{1} \in T_{1}^{\prime}$ and $\tau_{2} \in T_{2}^{\prime}$. Then it is easy to see that $\left\{A\left(z_{1}, z_{2}\right)\right\}^{-1}$ is analytic for all $\left(z_{1}, z_{2}\right)$ provided that the real parts of $z_{1}$ and $z_{2}$ belong to $T_{1}$ and $T_{2}$ respectively. ${ }^{(5)}$

In this and the next Sections we shall assume that the measure $\nu\left(u_{1}, u_{2}\right)$ is absolutely continuous with respect to the Lebesgue measure on $R_{2}$, that is, $U$ has a probability density $f\left(u_{1}, u_{2} ; \tau_{1}, \tau_{2}\right)$ which is written in the form

$$
f\left(u_{1}, u_{2} ; \tau_{1}, \tau_{2}\right)=A\left(\tau_{1}, \tau_{2}\right) e^{-\tau_{1} u_{1}-\tau_{2} u_{2}+\tau_{1}\left(u_{1}, u_{2}\right)},
$$

where the carrier $\mathfrak{D}\left(u_{1}, u_{2}\right)$ of $e^{\left.v i u_{1}, u_{2}\right)}$ is independent of $\tau_{1}$ and $\tau_{2}$.

Similarly to the one-parameter case, we have

Theorem 2. Let $U=\left(U_{1}, U_{2}\right)$ have the probability density of the form (6.4). Assume that the following conditions are satisfied:

(i) $\theta\left(z_{1}, z_{2}\right)$ is analytic in the cylinder $s_{2} \in T_{1}, s_{2} \in T_{2}$, where $z_{1}=s_{1}$ $+i t_{1}$ and $z_{2}=s_{2}+i t_{2}$.

$$
\int_{-\infty}^{\infty} \int_{-\infty}^{\infty}\left|\varphi_{s_{1}, s_{2}}\left(-t_{1},-t_{2}\right) \theta\left(s_{1}+i t_{1}, s_{2}+i t_{2}\right)\right| d t_{1} d t_{2}<\infty,
$$

for $s_{1} \in T_{1}, s_{2} \in T_{2}$, where $\varphi_{s_{1}, s_{2}}\left(t_{1}, t_{2}\right)$ is the characteristic function of the distribution of $U=\left(U_{1}, U_{2}\right)$, when the parameters $\tau_{1}=s_{1}$ and $\tau_{2}=s_{2}$.

$$
\lim _{t_{1}, t_{2} \rightarrow \infty} \varphi_{s_{1}, s_{2}}\left(-t_{1},-t_{2}\right) \theta\left(s_{1}+i t_{1}, s_{2}+i t\right)=0
$$

uniformly in every closed subdomain of $s_{1} \in T_{1}$ and $s_{2} \in T_{2}$.

Then if we put

$$
w\left(u_{1}, u_{2}\right)=\left(\frac{1}{2 \pi i}\right)^{2} \int_{s_{2}-i \infty}^{s_{2}+i \infty} \int_{s_{1}-i \infty}^{s_{1}+i \infty} \frac{\theta\left(z_{1}, z_{2}\right)}{A\left(z_{1}, z_{2}\right)} e^{z_{1} u_{1}+z_{2} u_{2}} d z_{1} d z_{2},
$$

for $s_{i} \in T_{i},\left(u_{i}, u_{2}\right) \in \mathfrak{D}\left(u_{1}, u_{2}\right), i=1,2$,

$$
\delta(U)=\left.e^{-v\left(u_{1}, u_{2}\right)} w\left(u_{1}, u_{2}\right)\right|_{\left(u_{1}, u_{2}\right)=\left(l_{1}, i_{2}\right)}
$$

is the unique unbiased sufficient estimate of $\theta\left(\tau_{1}, \tau_{2}\right)$.

15) Cf. Bochner-Martin [3], p. 140 
Further, if there exists a linear operator $A_{u_{1}, u_{2}}$ satisfying the condition

$$
A_{u_{1}, u_{2}} e^{\tau_{1} u_{1}+\tau_{22} u_{2}}=\theta\left(\tau_{1}, \tau_{2}\right) e^{\tau_{1} u_{1}+\tau_{2} u_{2}},
$$

and the existence of both members and equality

$$
\text { (v) } \begin{aligned}
\int_{-\infty}^{\infty} \int_{-\infty}^{\infty} A_{u_{1}, u_{2}}\{ & \left\{\frac{e^{\left(s_{1}+i t_{1}\right) u_{1}+\left(s_{2}+i t_{2}\right) u_{2}}}{A\left(s_{1}+i t_{1}, s_{2}+i t_{2}\right)}\right\} d t_{1} d t_{2} \\
& =A_{u_{1}, u_{2}}\left\{\int_{-\infty}^{\infty} \int_{-\infty}^{\infty} \frac{e^{\left(s_{1}+i t_{1}\right) u_{1}+\left(s_{2}+i t_{2}\right) u_{2}}}{A\left(s_{1}+i t_{1}, s_{2}+i t_{2}\right)} d t_{1} d t_{2}\right\}
\end{aligned}
$$

are affirmed, then the above estimate can be expressed in the form

$$
\delta(U)=\left.e^{-v\left(u_{1}, u_{2}\right)} \Lambda_{u_{1}, u_{2}} e^{v\left(u_{1}, u_{2}\right)}\right|_{\left(u_{1}, u_{2}\right)=\left(u_{1}, r_{2}\right)} .
$$

From this theorem we obtain

Corollary 2.1. Suppose that the sufficient statistic $U=\left(U_{1}, U_{2}\right)$ has the probability density of the form (6.4). Then the unique unbiased sufficient estimate $\delta\left(U_{1}, U_{2}\right)$ of

$$
\theta\left(\tau_{1}, \tau_{2}\right)=\tau_{1}^{k_{1}{ }^{1}} \tau_{2}^{k_{2}}
$$

(where $k_{1}$ and $k_{2}$ are both non-negative integers) is given by

$$
\delta\left(U_{1}, U_{2}\right)=\left.e^{-v\left(u_{1}, u_{2}\right)} \frac{\partial^{k_{1}} \partial^{k_{2}}}{\partial u_{1}^{k_{1}} \partial u_{2}^{k_{2}}} e^{v\left(u_{1}, u_{2}\right)}\right|_{\left(u_{1}, u_{2}\right)=\left(u_{1}, v_{2}\right)},
$$

provided that the conditions (ii) and (iii) of Theorem 2 are satisfied.

This result can be got similarly to Corollary 1.1 and the proof is omitted.

Corollary 2.2. Let the sufficient statistic $U=\left(U_{1}, U_{2}\right)$ have the probability density of the form (6.4), where

$$
\mathfrak{D}\left(u_{1}, u_{2}\right)=\left\{\left(u_{1}, u_{2}\right) ; K_{1}\left(u_{2}\right)<u_{1}<K_{2}\left(u_{2}\right),-\infty<u_{2}<\infty\right\},
$$

and $\tau_{1}>0$. Suppose further its density have $(k+1)$ continuous derivatives with respect to $u_{2}$ all belonging to class $L_{1}(-\infty, \infty)$.

Assume ihat

$$
\theta\left(\tau_{1}, \tau_{2}\right)=\tau_{1}^{-\alpha} \tau_{2}^{k}
$$

(where $\alpha$ is a positive real number and $k$ is a non-negative integer) together with the density function satisfies the conditions (ii) and (iii) of Theorem 2. Then the unique unbiased sufficient estimate $\delta\left(U_{1}, U_{2}\right)$ of $\theta\left(\tau_{1}, \tau_{2}\right)$ is given by

$$
\delta\left(U_{1}, U_{2}\right)=\left.e^{-v\left(u_{1}, u_{2}\right)} \frac{1}{\Gamma(\alpha)} \frac{\partial^{k}}{\partial u_{2}^{k}} \int_{K_{2}^{\prime}\left(u_{1}, u_{2}\right)}^{K_{1^{\prime}}\left(u_{1}, u_{2}\right)} x^{\alpha-1} e^{v\left(u_{1}-x_{1}, u_{2}\right)} d x\right|_{\left(u_{1}, u_{2}\right)=\left(r_{1}^{\prime}, r_{2}\right)},
$$

where $K_{i}^{\prime}\left(u_{1}, u_{2}\right)=\max \left(u_{1}+K_{i}\left(u_{2}\right), 0\right)$. 
Proof. We obtain by the properties of convolution,

$$
\begin{aligned}
& w\left(u_{1}, u_{2}\right) \\
& =\left(\frac{1}{2 \pi}\right)^{2} \frac{1}{A\left(s_{1}, s_{2}\right)} \int_{-\infty}^{\infty} \int_{-\infty}^{\infty}\left(s_{2}+i t_{2}\right)^{k} e^{\left(s_{1}+i t_{1}\right) u_{1}+\left(s_{2}+i t_{2}\right) u_{2}} \frac{\varphi_{s_{1}, s_{2}}\left(-t_{1},-t_{2}\right)}{\left(s_{1}+i t_{1}\right)^{\alpha}} d t_{1} d t_{2} \\
& =\frac{1}{\Gamma(\alpha)} \frac{\partial^{k}}{\partial u_{2}^{k}} \int_{K_{2}^{\prime}\left(u_{1}, u_{2}\right)}^{K_{1^{\prime}\left(u_{1}, u_{2}\right)}} x^{\alpha-1} e^{v\left(u_{1}-x, u_{2}\right)} d x .
\end{aligned}
$$

Hence the proof is completed by making use of Theorem 2 .

Corollary 2.3. Suppose that the assumptions made on $U=\left(U_{1}, U_{2}\right)$ in Corollary 2.2 are slightly changed so that the range of $\tau_{2}$ be $(0, \infty)$, instead of $(-\infty, \infty)$. Then if

$$
\theta\left(\tau_{1}, \tau_{2}\right)=\tau_{1}^{\alpha} \tau_{2}^{\beta}
$$

(where $\alpha$ and $\beta$ are assigned positive numbers) satisfies the conditions (ii) and (iii) of Theorem 2, the unique unbiased sufficient estimate $\delta\left(U_{1}\right.$, $\left.U_{2}\right)$ of $\theta\left(\tau_{1}, \tau_{2}\right)$ is given by

$$
\begin{aligned}
\delta\left(U_{1}, U_{2}\right)= & e^{-v\left(u_{1}, u_{2}\right)} \frac{1}{\Gamma(M-\alpha) \Gamma(N-\beta)} \frac{\partial^{M}}{\partial u_{1}{ }^{M}} \frac{\partial^{N}}{\partial u_{2}{ }^{N}} \\
& \left.\cdot \int_{0}^{\infty} \int_{K_{2}{ }^{\prime}\left(u_{1}, u_{2}\right)}^{K_{1}{ }^{\prime}\left(u_{1}, u_{2}\right)} x^{M-\alpha-1} y^{N-\beta-1} e^{v\left(u_{1}-x, u_{2}-y\right)} d x d y\right|_{\left(u_{1}, u_{2}\right)=\left(U_{1}, i_{2}\right)},
\end{aligned}
$$

where $M=[\alpha]+1, N=[\beta]+1$ and $K_{1}^{\prime}\left(u_{1}, u_{2}\right)$ and $K_{2}^{\prime}\left(u_{1}, u_{2}\right)$ are defined in the same way as in Corollary 2.2.

Proof. Similarly to the proof of Corollary 2.2, making use of convoluion properties, we get

$$
\begin{aligned}
w\left(u_{1}, u_{2}\right)= & \left(\frac{1}{2 \pi}\right)^{2} \frac{1}{A\left(s_{1}, s_{2}\right)} \int_{-\infty}^{\infty} \int_{-\infty}^{\infty}\left(s_{1}+i t_{1}\right)^{M}\left(s_{2}+i t_{2}\right)^{N} e^{\left(s_{1}+i t_{1}\right) u_{1}+\left(s_{2}+i t_{2}\right) u_{2}} \\
& \cdot \frac{\varphi_{s_{1}, s_{2}}\left(-t_{1},-t_{2}\right)}{\left(s_{1}+i t_{1}\right)^{M-\alpha}\left(s_{2}+i t_{2}\right)^{N-\beta}} d t_{1} d t_{2} \\
= & \frac{1}{\Gamma(M-\alpha) \Gamma(N-\beta)} \frac{\partial^{M}}{\partial u_{1}^{M}} \frac{\partial^{N}}{\partial u_{2}^{N}} \int_{K_{2^{\prime}}\left(u_{1}, u_{2}\right)}^{K_{1^{\prime}\left(u_{1}, u_{2}\right)}^{M-\alpha-1}} d x \int_{0}^{\infty} y^{N-\beta-1} e^{v\left(u_{1}-x, u_{2}-y\right)} d y .
\end{aligned}
$$

This relation together with Theorem 2 proves the corollary.

Making use of these corollaries, it is easy to treat the case where the values of $\alpha$ and $\beta$ are not restricted as above.

$\S$ 7. Applications (2): Example 3. In this Section the case of normal variable with unknown mean and variance is treated. Suppose that $X_{i}$ 's are distributed according to $N\left(a, \sigma^{2}\right)$, where $i=1,2, \ldots, n,-\propto<a<\infty$ and $\sigma^{2}>0$. 
Then the probabilidy density of $X=\left(X_{1}, X_{2}, \ldots, X_{n}\right)$ is as follows:

$$
\left(\frac{1}{\sqrt{2 \pi \sigma}}\right)^{n} e^{-\frac{n a^{2}}{2 \sigma^{2}}} e^{-\frac{1}{2 \sigma^{2}}\left(\sum_{i=1}^{n} x_{i}{ }^{2}\right)-\frac{a}{\sigma^{2}-1}\left(-\sum_{i=1}^{n} x_{i}\right)} \text {. }
$$

Putting $\tau_{1}=\frac{1}{2 \sigma^{2}}, \tau_{2}=\frac{a}{\sigma^{2}}, u_{1}(x)=\sum_{i=1}^{n} x_{i}^{2}$ and $u_{2}(x)=-\sum_{i=1}^{n} x_{i}$, it is clear that $U=\left(U_{1}, U_{2}\right)=\left(u_{1}(X), u_{2}(X)\right)$ is the sufficient statistic for this class of distributions and its probability density is

$$
\frac{n^{\frac{n}{2}-2} e^{-\frac{n \tau_{2} 2}{4 \tau_{1}}} \tau_{1}^{\frac{n}{2}}}{\sqrt{\pi} \Gamma\left(\frac{n-1}{2}\right)} e^{-\tau_{1} u_{1}-\tau_{i} u_{2}}\left\{1\left(u_{1}-\frac{u_{2}^{2}}{n}\right)\right\}^{\frac{n-1}{2}-1},
$$

where $\boldsymbol{u}_{2}^{2} / n<\boldsymbol{u}_{1}<\infty,-\infty<\boldsymbol{u}_{2}<\infty, \boldsymbol{K}_{1}\left(\boldsymbol{u}_{2}\right)=\boldsymbol{u}_{2}^{2} / \boldsymbol{n}$ and $\boldsymbol{K}_{2}\left(\boldsymbol{u}_{2}\right)=\infty$.

Problem 3.1. Suppose that

$$
\theta\left(\tau_{1}, \tau_{2}\right)=\frac{\tau_{1}^{-1} \tau_{2}^{0}}{2}\left(=\sigma^{2}\right)
$$

To this problem Corollary 2.2 is to be applied. From (6.9)

$$
\begin{aligned}
\delta\left(u_{1}, u_{2}\right) & =\frac{1}{2\left\{\frac{1}{n}\left(u_{1}-\frac{u_{2}}{n}\right)^{2}\right\}^{n-1} \frac{1}{2}-1} \int_{0}^{u_{1}-\frac{u_{2} 2^{2}}{n}}\left(u_{1}-x-\frac{u_{2}^{2}}{n}\right)^{\frac{n-1}{2}-1} d x \\
& =\frac{1}{2}\left(u_{1}-\frac{u_{2}^{2}}{n}\right) .
\end{aligned}
$$

Therefore

$$
\delta\left(U_{1}, U_{2}\right)=\frac{\sum_{i=1}^{n}\left(X_{i}-\bar{X}\right)^{2}}{n-1}
$$

is the required unbiased estimate.

Problem 3.2. Suppose that

$$
\theta\left(\tau_{1}, \tau_{2}\right)=\tau_{1}^{-1} \tau_{2} / 2(=a) .
$$

Corollary 2.2 is also applicable to this problem. From (6.9)

$$
\begin{aligned}
\delta\left(u_{1}, u_{2}\right) & =\frac{1}{2\left\{\frac{1}{n}\left(u_{1}-\frac{u_{2}^{2}}{n}\right)\right\}^{\frac{n-1}{2}-1}} \frac{\partial}{\partial u_{2}} \int_{0}^{u_{1}-\frac{u_{2}{ }^{2}}{n}} \frac{1}{n}\left(u_{1}-y-\frac{u_{2}^{2}}{n}\right)^{\frac{n-1}{2}-1} d y \\
& =\frac{1}{n} u_{2} .
\end{aligned}
$$

Therefore,

$$
\delta\left(U_{1}, U_{2}\right)=\bar{X}
$$

is the required estimate. 
Problem 3.3. Suppose that

$$
\theta\left(\tau_{1}, \tau_{2}\right)=\tau_{1}^{-1 / 2} \tau_{2} / 2 \quad(=a / \sigma) .
$$

From (6.9) of Corollary 2.2

$$
\begin{aligned}
\delta\left(u_{1}, u_{2}\right) & \left.=\frac{1}{2\left\{\frac{1}{n}\left(u_{1}-\frac{u_{2}^{2}}{n}\right)\right\}^{\frac{n-1}{2}-1}} \frac{1}{\Gamma\left(\frac{1}{2}\right)} \frac{\partial}{\partial u_{2}} \int_{1}^{u_{1}-\frac{u_{2} 2}{n}} x^{-\frac{1}{2}\left\{\frac{1}{n}\left(u_{1}-x-\frac{u_{2}}{n}\right)\right.}\right\}^{n-1-1} d x \\
& =\frac{\Gamma\left(\frac{n-1}{2}\right)}{2 \Gamma\left(\frac{n-1}{2}-\frac{1}{2}\right)}\left(u_{1}-\frac{u_{2}^{2}}{2}\right)^{-\frac{1}{2}}\left(-\frac{2 u_{2}}{n}\right) .
\end{aligned}
$$

Therefore

$$
\delta\left(U_{1}, U_{2}\right)=\frac{\Gamma\left(\frac{n-1}{2}\right)}{\Gamma\left(\frac{n-1}{2}-\frac{1}{2}\right)} \cdot \frac{\bar{X}}{\sqrt{\sum_{i=1}^{n}\left(X_{i}-X\right)^{2}}} .
$$

Problem 3.4. Suppose that

$$
\theta\left(\tau_{1}, \tau_{2}\right)=\left(\frac{1}{\sqrt{2 \pi \tau_{1}}} \int_{A} e^{-\tau_{1}\left(x-\frac{\tau_{2}}{2 \tau_{1}}\right)^{2}} d y\right)^{k},
$$

where $A$ is a subset of $R_{1}$ and $k$ is an assigned positive integer. This problem has as same meaning as the latter part of Problem 1.3 of Example 1 in Section 4.

Here the conditions of Theorem 2 are satisfied. And

$$
\begin{aligned}
& w\left(u_{1}, u_{2}\right)=\left(\frac{1}{v^{\prime} 2 \pi}\right)^{2} \int_{-\infty}^{\infty} \int_{-\infty}^{\infty} e^{\left(s_{1}+i t_{1}\right) u_{1}+\left(s_{2}+i t_{2}\right) u_{2}} \cdot \frac{1}{A\left(s_{1}+i t_{1}, s_{2}+i t_{2}\right)}
\end{aligned}
$$

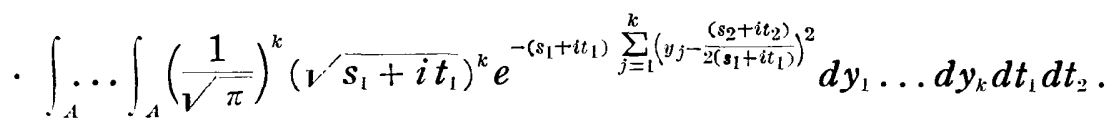

But

$$
A\left(s_{1}+i t_{1}, s_{2}+i t_{2}\right)=\frac{n^{\frac{n-4}{2}}}{\sqrt{\pi} \Gamma\left(\frac{n-1}{2}\right)}\left(s_{1}+i t_{1}\right)^{\frac{n}{2}} e^{-\frac{n\left(s_{2}+i t_{2}\right)^{2}}{f\left(s_{1}+i t_{1}\right)}} .
$$

Therefore

$$
\begin{aligned}
& w\left(u_{1}, u_{2}\right)=\left(\frac{1}{2 \pi}\right)^{2}\left(\frac{1}{\sqrt{\pi}}\right)^{k} \frac{1}{C_{n}} \int_{A} \ldots \int_{A} d y_{1} \ldots d y_{k} \\
& \text { - } \int_{-\infty}^{\infty} \int_{-\infty}^{\infty} e^{\left(s_{1}+i t_{1}\right) u_{1}+\left(s_{2}+i t_{2}\right) u_{2}}\left(\frac{1}{\sqrt{s_{1}+i t_{1}}}\right)^{n-k}
\end{aligned}
$$

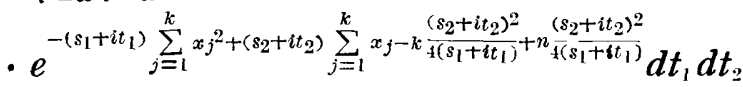

$$
\begin{aligned}
& =\left(\frac{1}{2 \pi}\right)^{2} \frac{1}{C_{n}}\left(\frac{1}{\sqrt{ } \pi}\right)^{k} \int_{A} \ldots \int_{A} d y_{1} \ldots d y_{k} \times I_{1} \text {, }
\end{aligned}
$$


where

$$
\begin{aligned}
& I_{1}=\int_{-\infty}^{\infty}\left(\frac{1}{V}\right)^{n-k} e^{\left(s_{1}+i t_{1}\right) u_{1}-\left(s_{1}+i t_{1}\right)} \sum_{j=1}^{k} y_{j 2}^{2} d t_{1} \times I_{2}, \\
& I_{2}=\int_{-\infty}^{\infty} e^{\left(s_{2}+i t \overline{\mathrm{g}}\right) u_{2}+\left(s_{2}+i t_{2}\right)} \sum_{j=1}^{k} y_{j}+\frac{(n-k)\left(s_{2}+i t_{2}\right)^{2}}{4\left(s_{1}+i t_{1}\right)} d t_{2}
\end{aligned}
$$

and

$$
C_{n}=\frac{n^{\frac{n-4}{2}}}{\sqrt{\pi} \Gamma\left(\frac{n-1}{2}\right)} .
$$

By making use of properties of characteristic function of normal distribution we have

$$
I_{2}=\frac{\sqrt{4 \pi}}{\sqrt{n-k}} e^{-\frac{\left(s_{1}+i t_{1}\right)}{n-k}\left(u_{2}+\sum_{j=1}^{k} y_{j}\right)^{2}} .
$$

Hence by making use of properties of characteristic function of gamma distribution we have

$$
\begin{aligned}
I_{1} & =\frac{\sqrt{4 \pi}}{\sqrt{n-k}} e^{s_{1}\left\{u_{1}-\sum_{j=1}^{k} y_{j^{2}-}-\frac{\left(u_{2}+\sum_{j=1}^{k} y_{j}\right)^{2}}{n-k}\right\}} \int_{-\infty}^{\infty}\left(\frac{1}{\sqrt{s_{1}+i t}}\right)^{\frac{n-k-1}{2}} e^{i t_{1}\left\{u_{1}-\sum_{j=1}^{k} y_{j^{2}}-\frac{\left(u_{2}+\sum_{j=1}^{k} y_{j}\right)^{2}}{n-k}\right\}} d t \\
& =\left\{\begin{array}{c}
\frac{\sqrt{4 \pi}}{\sqrt{n-k}} 2 \pi \frac{1}{\Gamma\left(\frac{n-k-1}{2}\right)}\left\{u_{1}-\sum_{j=1}^{k} y_{j}^{2}-\frac{1}{n-k}\left(u_{2}+\sum_{j=1}^{k} y_{j}\right)^{2}\right\} \\
\text { for }\left\{u_{1}-\sum_{j=1}^{k} y_{j}^{2}-\frac{1}{n-k}\left(u_{2}+\sum_{j=1}^{k} y_{j}\right)^{2}\right\} \geq 0 \\
\text { otherwise. }
\end{array}\right.
\end{aligned}
$$

Hence

$$
\begin{aligned}
& w\left(u_{1}, u_{2}\right)=\left\{\begin{array}{r}
\int_{A} \ldots \int_{A}\left(\frac{1}{\sqrt{\pi}}\right)^{k} \frac{\Gamma\left(\frac{n-1}{2}\right)}{\Gamma\left(\frac{n-k-1}{2}\right)^{2}} \frac{1}{\sqrt{n-4}} \frac{1}{n^{\frac{n-4}{2}}}\left\{u_{1}-\sum_{j=1}^{k} y_{j}^{2}\right. \\
\left.-\frac{1}{n-k}\left(u_{2}+\sum_{j=1}^{k} y_{j}\right)^{2}\right\}^{\frac{n-k-1}{2}-1}
\end{array}\right. \\
& \text { for } \quad\left\{u_{1}-\sum_{j=1}^{k} y_{j}^{2}-\frac{1}{n-k}\left(u_{2}+\sum_{j=1}^{k} y_{j}\right)^{2}\right\} \geq 0 \\
& 0 \text { otherwise. }
\end{aligned}
$$

Now we put

$$
\delta\left(u_{1}, u_{2}\right)=\int_{A} \ldots \int_{d} \varphi\left(y_{1}, y_{2}, \ldots, y_{k}\right) d y_{1} d y_{2}, \ldots d y_{k} .
$$


Then we have

where

$$
\left\{\begin{aligned}
&\left(\frac{1}{\sqrt{\pi}}\right)^{k} \frac{\Gamma\left(\frac{n-1}{2}\right)}{\Gamma\left(\frac{n-k-1}{2}\right)} \frac{1}{\sqrt{n-k}} \frac{1}{n^{\frac{k}{2}-\frac{1}{2}}} \\
& \frac{\left\{\frac{1}{n \sigma^{\prime 2}}\left[u_{1}-\sum_{j=1}^{k} y_{j}^{2}-\frac{1}{n-k}\left(u_{2}+\sum_{j=1}^{k} y_{j}\right)^{2}\right]\right\}^{\frac{n-k-1}{2}-1}}{\sigma^{\prime k}} \\
& \text { for }\left[u_{1}-\sum_{j=1}^{k} y_{j}{ }^{2}-\frac{1}{n-k}\left(u_{2}+\sum_{j=1}^{k} y_{j}\right)\right] \geq 0 \\
& 0 \quad \text { otherwise, }
\end{aligned}\right.
$$

$$
\sigma^{\prime 2}=\frac{1}{n}\left(u_{1}-\frac{u_{2}^{2}}{n}\right)
$$

On the other hand we have,

where

$$
\begin{aligned}
\frac{1}{n \sigma^{\prime 2}}\left[u_{1}-\sum_{j=1}^{k} y_{j}{ }^{2}-\frac{1}{n-k}\left(u_{i}+\sum_{j=1}^{k} y_{j}\right)^{2}\right] \\
=1-\begin{array}{c}
n-k+1 \\
\sigma^{\prime 2} n(n-k)
\end{array}\left\{\sum_{j=1}^{k} \sum_{i=1}^{k} a_{i j}\left(y_{i}+\frac{u_{i j}}{n}\right)\left(y_{j}+\frac{u_{i}}{n}\right)\right\},
\end{aligned}
$$

Therefore

$$
a_{i j}=\left\{\begin{array}{cl}
1 & \text { for } \quad i=j \\
\frac{1}{n-k+1} & \text { for } i \neq j .
\end{array}\right.
$$

$$
\varphi\left(y_{1}, y_{2}, \ldots, y_{k}\right)=\left\{\begin{array}{l}
\frac{\left[1-\frac{n-k+1}{n(n-k) \sigma^{\prime 2}} \sum_{i=1}^{k} \sum_{j=1}^{k} a_{i j}\left(y_{i}+\frac{u_{2}}{n}\right)\left(y_{j}+\frac{u_{i 2}}{n}\right)\right]^{\frac{n-k-1}{2}-1}}{\sigma^{\prime k}} \\
\text { for } \frac{\sum_{i=1}^{k} \sum_{j=1}^{k} a_{i j}\left(y_{i}+\frac{u_{2}}{n}\right)\left(y_{j}+\frac{u_{2}}{n}\right)}{\sigma^{\prime k}} \leqq \frac{n(n-k)}{n-k+1} \\
0 \quad \text { otherwise. }
\end{array}\right.
$$

Introducing the function

$$
f_{n}\left(t_{1}, t_{2}, \ldots, t_{k}\right)=\left\{\begin{array}{c}
C_{n}{ }^{\prime}\left[1-\frac{n-k+1}{n(n-k)} \sum_{j=1}^{k} \sum_{i=1}^{k} a_{i j} t_{i} t_{j}\right] \\
\text { for } \sum_{j=1}^{k} \sum_{i=1}^{k} a_{i j} t_{i} t_{j} \leq \frac{n(n-k)}{n-k+1} \\
0 \quad \text { otherwise, }
\end{array}\right.
$$


where

$$
\begin{gathered}
t_{i}=\left(y_{i}+\frac{u_{2}}{n}\right) / \sigma^{\prime} \\
C_{n}{ }^{\prime}=\left(\frac{1}{\sqrt{\pi}}\right)^{k} \frac{\Gamma\left(\frac{n-1}{2}\right)}{\Gamma\left(\frac{n-k-1}{2}\right)^{\sqrt{n-k}}} \frac{1}{n^{\frac{k}{2-\frac{1}{2}}}},
\end{gathered}
$$

and

we can write $\varphi\left(y_{1}, y_{2}, \ldots, y_{k}\right)$ in the form

$$
\varphi\left(y_{1}, y_{2}, \ldots, y_{k}\right)=\left(\frac{1}{\sigma^{\prime}}\right)^{k} f_{n}\left(t_{1}, t_{2}, \ldots, t_{k}\right) .
$$

As $n \rightarrow \infty$, the function $f_{n}$ converges to the limit

$$
f_{\infty}\left(t_{1}, t_{2}, \ldots, t_{k}\right)=\left(\frac{1}{\sqrt{2 \pi}}\right)^{k} e^{-\frac{1}{2} \sum_{i=1}^{k} t_{i}^{2}} .
$$

This result coincides with the KOLMOGOROFF's one in the case where $k=1$.

\$ 8 . The discrete case. The discrete case can be treated similarly to the previous Sections by utilizing the concepts about Laplace-Stieltjes transforms. density

Let the sufficient statistic $U=\boldsymbol{u}(X)$ have the generalized probability

$$
f(u ; \tau)=A(\tau) e^{-\tau u+v i u)} \quad u \in \mathscr{D}(u)
$$

satisfying the conditions as mentioned in Section 3 , and $\mathfrak{D}(\boldsymbol{u})$ be a discrete subset of $R_{1}$ which are bounded to right or left hand and independent of $\tau$. In what follows we shall assume that $\mathfrak{D}(\boldsymbol{u})$ is a set of non-negative integers without loss of generality. Further, assume that

$$
\{A(\tau)\}^{-1}=\int_{0}^{\infty} e^{-\tau u^{\prime}} d\left\{\sum_{u^{\prime}=0}^{\left[u^{\prime}\right]} e^{v\left(u^{\prime \prime}\right)}\right\}<\infty .
$$

Then the following theorem holds:

Theorem 3. Assume that for a given parametric function $\theta(\tau)$ there cxists an unbiased estimate; i.e, an estimate $\delta(U)$ such that

$$
\theta(\tau)=A(\tau) \int_{0}^{\infty} e^{-\tau u^{\prime}} d\left\{\sum_{u^{\prime}=0}^{\left[u^{\prime}\right]} \delta\left(u^{\prime \prime}\right) e^{v\left(u^{\prime \prime}\right)}\right\},
$$

where $\left.\sum_{u^{\prime}=0}^{u} \delta\left(u^{\prime}\right) e^{v} u^{\prime}\right)$ is of bounded variation in $\left(0, u_{0}\right)$ for every positive $u_{0}$. Then $\delta(U)$ is the unique unbiased sufficient estimate of $\theta(\tau)$ and given by the formula

$$
\delta(U)=\left.2 e^{-v(u)}(-1)^{u} \sum_{k=0}^{n}(-1)^{k}\left\{\delta(k) e^{n(k)}\right\}^{*}\right|_{u=v},
$$


where

$$
\left\{e^{v(u)} \delta(u)\right\}^{*}=\frac{1}{2 \pi i}\left\{\int_{s-i \infty}^{s+i \infty} \frac{\theta(z)\left\{e^{u z}-e^{(u-1) z}\right\}}{z} \frac{1}{A} \frac{1}{(z)} d z\right\}, \quad \text { for any } s \in T .
$$

Further, suppose that there exists a linear operator satisfying the condition

$$
\Lambda_{u}\left\{e^{u z}\right\}=\theta(z) e^{z u}, \quad \text { for } \Re(z) \in T,
$$

and the existence and equality of both sides of

(ii) $\frac{1}{2 \pi i} \int_{s-i \infty}^{s+i \infty} \frac{A_{u}\left\{e^{z u}\right\}}{z}\left\{\sum_{u^{\prime}=0}^{\infty} e^{v\left(w^{\prime}\right)} e^{-z u^{\prime}}\right\} d z=\frac{1}{2 \pi i} A_{u}\left\{\int_{s-i \infty}^{s+i \infty} \frac{e^{z u}}{z}\left\{\sum_{u^{\prime}=0}^{\infty} e^{v\left(u^{\prime}\right)} e^{-z u^{\prime}}\right\} d z\right\}$ are affirmed. Then $\delta(U)$ is expressed in the form

$$
\delta(U)=\left.e^{-v i u)} A_{u}\left\{e^{v(u)}\right\}\right|_{u=i}
$$

Proof. If an unbiased sufficient estimate of $\theta(\tau)$ exists, the uniqueness follows from the completeness of our family of distributions. ${ }^{16)}$

Now let us put

$$
\left\{e^{v(u)} \delta(u)\right\}^{*}=\left\{\begin{array}{cr}
e^{v(u)} \delta(u) & u \leqq 0 \\
\frac{\delta(u) e^{v(u)}+\delta(u-1) e^{v(u-1)}}{2} & \infty>u>0 \\
e^{v(u)} \delta(u) & u=\infty
\end{array}\right.
$$

Then utilizing the theorem concerning Laplace transforms we have ${ }^{(7)}$

$$
\begin{aligned}
\left\{\delta(u) e^{v(u)}\right\}^{*} & =\frac{1}{2 \pi i}\left\{\int_{s-i \infty}^{s+i \infty} \frac{\theta(z) e^{u z}}{z} \frac{1}{A(z)} d z-\int_{s-i \infty}^{s+i \infty} \frac{\theta(z) e^{(u-1) z}}{z} \frac{1}{A(z)} d z\right\} \\
& =\frac{1}{2 \pi i}\left\{\int_{s-i \infty}^{s+i \infty} \frac{\theta(z)\left\{e^{u z}-e^{(u-1) z}\right\}}{z} \frac{1}{A(z)} d z\right\}, \quad \text { for } s \in T .
\end{aligned}
$$

So that the definition of $\left\{\delta(u) e^{v(u)}\right\} *$ furnishes the first half of the theorem.

If we use (i) and (ii),

$$
\begin{aligned}
\sum_{u^{\prime}=0}^{u}\left\{\delta\left(u^{\prime}\right) e^{v\left(u^{\prime}\right)}\right\}^{*} & =\frac{1}{2 \pi i} \int_{s-i \infty}^{s+i \infty} \frac{\Lambda_{u}\left\{e^{z u}\right\}}{z}\left\{\sum_{u^{\prime}=0}^{\infty} e^{v\left(u^{\prime}\right)} e^{-z u^{\prime}}\right\} d z \\
& =\frac{1}{2 \pi i} \Lambda_{u}\left[\sum_{u^{\prime}=0}^{\infty} e^{v\left(u^{\prime}\right)} \int_{s-i \infty}^{s+i \infty} \frac{e^{z\left(u-u^{\prime}\right)}}{z} d z\right] .
\end{aligned}
$$

16) With respect to the notion of completencss, sce LeHMAn-SoHefré [14].

17) Cf. Widder [20] p. 69. 
But

$$
\frac{1}{2 \pi i} \int_{s-i \infty}^{s+i \infty} \frac{e^{z\left(u-u^{\prime}\right)}}{z} d z= \begin{cases}0 & u^{\prime}>u \\ \frac{1}{2} & u^{\prime}=u \\ 1 & u^{\prime}<u\end{cases}
$$

Hence

$$
\sum_{u^{\prime}=0}^{u}\left\{\delta\left(u^{\prime}\right) e^{v\left(u^{\prime}\right)}\right\}^{*}=A_{u}\left\{\sum_{u^{\prime}=0}^{u-1} e^{v\left(u^{\prime}\right)}+\frac{1}{2} e^{v(u)}\right\} .
$$

And

$$
\left\{\delta(u) e^{v(u)}\right\}^{*}=\Lambda_{u}\left\{\frac{1}{2}\left[e^{v(u)}+e^{v(u-1)}\right]\right\}=\Lambda_{u}\left\{e^{v *(u)}\right\},
$$

where

$$
e^{v *(u)}=\left\{\begin{array}{cr}
e^{v(u)} & u \leqq 0 \\
\frac{e^{v(u)}+e^{v(u-1)}}{2} & 0<u<\infty \\
e^{v(u)} & u=\infty .
\end{array}\right.
$$

So that we have by the definition of $\left\{\delta(u) e^{v i u)}\right\}^{*}$

namely,

$$
\begin{gathered}
\delta(u) e^{v i u)}=\Lambda_{u}\left[e^{v i u)}\right], \\
\delta(U)=e^{-v(u)} \Lambda_{u}\left[e^{v(u)}\right]_{u=c} .
\end{gathered}
$$

It should be noted that the existence or non-existence of an unbiased estimate depends on the behaviours of $A(\tau)$ and $\theta(\tau)$ in question. For example: $:^{\text {is }}$

(a) if $\psi(z)=\theta(z) / A(z)$ is analytic at every $z$ such that $\Re(z) \in T$ and if for some real $s$ in $T$ the two sequences

and

$$
\begin{array}{lll}
\left\{(-1)^{n}\right. & \left.\psi^{(n)}\left(s_{0}\right)\right\} & \boldsymbol{n}=0,1,2, \ldots \text { ad inf. } \\
\left\{(-1)^{n}\right. & \left.\psi^{(n)}\left(s_{0}\right)\right\} & \boldsymbol{n}=1,2,3, \ldots \text { ad inf. }
\end{array}
$$

are positive definite or positive semidefinite, or

(b) if $\psi(z)$ can be written in the form $\psi(z)=\psi_{1}(z)-\psi_{2}(z)$, by two functions $\psi_{1}(z)$ and $\psi_{2}(z)$ satisfying the condition (a), existence of such an estimate is affirmed.

(c) if $\psi(\tau)$ has derivatives of all orders in $0<\tau<\infty$ and there exists a constant $M$ such that

$$
\int_{0}^{\infty}\left|\psi^{(k)}(z)\right| \frac{z^{k-1}}{(k-1) !} d z<M \quad(k=1,2, \ldots) .
$$

When $\mathfrak{D}(u)$ is unbounded to both sides, e.g., $\mathscr{D}(u)=\{-\infty, \ldots,-2,-1$, $0,1,2, \ldots, \infty\}$, the above conditions must be slightly modified but the estimation problem can be treated quite similarly to the previous lines by making use of the properties of bilateral Laplace transforms.

Now we will give some illustrations about the discrete distributions. ${ }^{19}$

18) Cf. Widder [20] pp. 265-270, 306.

19) The idea of transformations is due to Prof. T. Kitagawa. 
Example 4. Poisson distribution. When $X_{i}^{\prime}$ ' $(i=1,2, \ldots, n)$ are distributed independently according to the Poisson distribution with a common unknown mean, $0<\lambda<\infty$, the probability distribution of $X=\left(X_{1}\right.$, $\left.X_{2}, \ldots, X_{n}\right)$ is

$$
\frac{e^{-n \lambda}}{x_{1} ! x_{2} ! \ldots x_{n} !} e^{\sum_{i=1}^{n} x_{i} \log \lambda}
$$

so that $U=\sum_{i=1}^{n} X_{i}$ is a sufficient statistic for this class of distributions and its probability distribution is

where $\tau=\log \frac{1}{\lambda}$.

$$
\left\{\begin{array}{cc}
e^{-n e^{-\tau}} \cdot e^{-u \tau+\log \frac{n^{u}}{u !}} & u \geq 0 \\
0 & u<0
\end{array}\right.
$$

Suppose that we wish to estimate $e^{-h \tau}$, that is, $\lambda^{h}$. In this case, making use of the operator $\Lambda_{u}^{(h)}$ such that

we obtain the estimate

$$
A_{u}^{(h)}\left[e^{\tau u}\right]=e^{-h \tau} e^{\tau u}=e^{\tau(n-h)},
$$

$$
\delta(U)=\left\{\begin{array}{cc}
\frac{U(U-1) \ldots(U-h+1)}{n^{h}} & U \geq h \\
0 & U<h .
\end{array}\right.
$$

Example 5. Binomial distribution. When $X_{i}$ 's $(i=1,2, \ldots, n)$ are mutually independent random variables which are identically distributed according to a binomial distribution with an unknown parameter $p$. Then the probability distribution of $X=\left(X_{1}, X_{2}, \ldots, X_{n}\right)$ is

$$
\prod_{i=1}^{n} p^{x_{i}}(1-p)^{1-x_{i}}=(1-p) e^{\sum_{i=1}^{n} x_{i} \log \frac{p}{1-p}} \text {. }
$$

Hence $U=\sum_{i=1}^{n} X_{i}$ is a sufficient statistic for this class of distributions and the probability distribution of $U$ is

$$
{ }_{n} C_{n}\left\{1+\frac{1}{1+e^{-\tau}}\right\}^{n} e^{-\tau u},
$$

where $\tau=\log \frac{p}{1-p},-\infty<\tau<0$.

For $e^{-\tau}(=p / q)$, making use of the operator $A_{u}$ such that

$$
\Lambda_{u}\left\{e^{u \tau}\right\}=e^{-\tau} e^{u \tau}=e^{\tau(u-1)},
$$

we get the unique unbiased sufficient estimate

$$
\delta(U)=U^{U}-{ }^{\prime} .
$$


And for $\sum_{k=0}^{\infty}(-1)^{k} \frac{(h+k) !}{k !} e^{-(h+k) r}\left(=p^{h}\right)$, we get the estimate

$$
\delta(U)=\left\{\begin{array}{ccc}
\sum_{k=0}^{u-h}(-1)^{k \cdot k(h+k) !}{ }_{n} C_{n-h-k} & \\
k ! & { }_{n} C_{u} & U \geqq h \\
0 & & U<h .
\end{array}\right.
$$

\section{KyUShu University, TOKYo University and KYUSHU UNIVERSITY.}

\section{References}

[1] D. BLACKWELL; Conditional expectation and unbiased sequential eslimation. Ann. Math. Stat., 18 (1947), 105-112.

[2] D. Brackwell and M. A. Girshick; Theory of games and statistical decisions. New York. 1954.

[3] S. Bochner and W. T. Martin; Several complex variables. Princeton. 1948.

[4] H. Cramér; Mathematical methods of statistics. Princeton. 1946.

[5] J. L. Dоов; Statistical estimation. Trans. Amer. Math. Soc., 39 (1936), 410-421.

[6] A. Dvoretzky, A. WALD and J. Wolfowitz; Elimination of randomization in certain statistical decision procedures and zero-sum two-person games. Ann. Math. Stat., 22 (1951), 1-21.

[7] R. A. FISHER; On the mathematical foundations of theoretical statistics. Phil. Trans. Roy. Soc. London., Ser. A. 222 (1922), 309-368.

[8] M. A. GIRshick and L. J. SAVAGE; Bayes and minimax estimates for quadratic loss functions. Proc. Second Berkeley Symp. on Math. Stat. and Prob., Berkeley and Los Angels 1951, $53-73$.

[9] P. R. HaLnos and L. J. SAVAGE; Applications of the Radon-Nykodim theorem to the theory of sufficient statistics. Ann. Math. Stat., 20 (1949), 225-241.

[10] P. Hoel; Conditional expectation and the efficiency of estimates. Ann. Math. Stat., 22 (1951), 299-301.

[11] T. KitAgAwa; The operational calculus and the estimations of functions of parameter admitting sufficient statistics. Bull. Math. Stat, 6 (1956), 95-108.

[12] A. N. Kolmogoroff; Unbiased estimates. Amer. Math. Soc. Translation, No. 98 (1953). (English translation).

[13] B. O. Koopman; On distributions admitting a sufficient statistics. Trans. Amer. Math. Soc., 39 (1936), $399-409$.

[14] E. L. Lehmann and H. SChefFé; Completeness, similar regions and unbiased estimation. Part I. Sankhyā, 10 (1950), 305 -340., Part II. Sankhyā, 15 (1955), 219 - 236.

[15] E. J. G. Pitman; Sufficient statistics and intrinsic accuracy. Proc. Camb. Phil. Soc., 32 (1936), $567-579$.

[16] C. R. RAO; Information and accuracy attainable in the estimation of statistical parameters. Bull. Calcutta Math. Soc., 37 (1945), 81-91.

[17] C. R. RAO; Sufficient statistics and minimum variance estimates. Proc. Camb. Phil. Soc., 45 (1949), $213-218$.

[18] E. SVERDRUP; Similarity, unbiassedness, minimaxibility and admissibility of statistical test procedures. Skandinavisk Aktuarietidskrift., 36 (1935), 64-86.

[19] A. WALD; Statistical decision functions. New York. 1950.

[20] D. V. WIDder; The Laplace transforms. Princeton. 1946.

[21] A. Zygmund; Trigonometrical series. Warszawa and Lwow. 1935. 